\title{
Factors associated with time delay to carotid stenting in patients with a symptomatic carotid artery stenosis
}

\author{
Klaus Gröschel · Sonja Schnaudigel · Katrin Wasser • \\ Sara M. Pilgram-Pastor • Ulrike Ernemann · \\ Michael Knauth · Andreas Kastrup
}

Received: 6 December 2010/Revised: 2 January 2011/ Accepted: 10 January 2011/Published online: 25 January 2011

(C) The Author(s) 2011. This article is published with open access at Springerlink.com

\begin{abstract}
Treatment of a symptomatic stenosis is known to be most beneficial within 14 days after the presenting event but this can frequently not be achieved in daily practice. The aim of this study was the assessment of factors responsible for this time delay to treatment. A retrospective analysis of a prospective two-center CAS database was carried out to investigate the potential factors that influence a delayed CAS treatment. Of 374 patients with a symptomatic carotid stenosis, $59.1 \%$ were treated beyond $\geq 14$ days. A retinal TIA event $(\mathrm{OR}=3.59,95 \%$ CI $1.47-8.74, p<0.01)$ was found to be a predictor for a delayed treatment, whereas the year of the intervention $(\mathrm{OR}=0.32,95 \%$ CI $0.20-0.50, p<0.01)$ and a contralateral carotid occlusion (OR $=0.42,95 \% \mathrm{CI}$ $0.21-0.86, p=0.02$ ) were predictive of an early treatment. Similarly, within the subgroup of patients with transient symptoms, the year of the intervention $(\mathrm{OR}=0.28,95 \% \mathrm{CI}$
\end{abstract}

K. Gröschel and S. Schnaudigel contributed equally to this manuscript.

K. Gröschel $(\bowtie) \cdot$ S. Schnaudigel · K. Wasser · A. Kastrup Department of Neurology, University of Göttingen, Robert-Koch-Str. 40, 37075 Göttingen, Germany e-mail: klaus.groeschel@medizin.uni-goettingen.de

K. Gröschel · A. Kastrup

Department of General Neurology, Center of Neurology

and Hertie-Institute for Clinical Brain Research,

University of Tübingen, Hoppe-Seyler-Strasse 3,

72076 Tübingen, Germany

S. M. Pilgram-Pastor · M. Knauth

Department of Neuroradiology, University of Göttingen,

Robert-Koch-Str. 40, 37075 Göttingen, Germany

U. Ernemann

Department of Neuroradiology, University of Tübingen,

Hoppe-Seyler-Strasse 3, 72076 Tübingen, Germany
$0.14-0.59, p<0.01)$ was associated with an early treatment, whereas a retinal TIA as the qualifying event $(\mathrm{OR}=6.96$, 95\% CI 2.37-20.47, $p<0.01$ ) was associated with a delayed treatment. Treatment delay was most pronounced in patients with an amaurosis fugax, whereas a contralateral carotid occlusion led to an early intervention. Although CAS is increasingly performed faster in the last years, there is still scope for an even more accelerated treatment strategy, which might prevent future recurrent strokes prior to treatment.

Keywords Symptomatic carotid stenosis $\cdot$ Stent $\cdot$ Stroke . Risk factors - Transient ischemic attack $\cdot \mathrm{ABCD}^{2}$ score Carotid artery stenting

\section{Introduction}

In the past few years, several population-based studies have clearly demonstrated that patients with a transient ischemic attack (TIA) or stroke have a high risk for a subsequent stroke [6]. Based on a six or seven-point score, using age, blood pressure, diabetes mellitus, clinical features, and duration of symptoms, the risk of further ischemic symptoms appears to be highly predictable, ranging from 3.1 to $17.8 \%$ at 90 days (ABCD and $\mathrm{ABCD}^{2}$ score) $[11,17]$. In addition, evidence has accumulated that TIA or mild-stroke patients with a large-artery atherosclerosis have a particularly high risk of early recurrent stroke, which reaches up to $28 \%$ within 14 days $[14,15]$. Moreover, an early carotid endarterectomy (CEA) has been shown to be associated with a beneficial outcome in contrast to an intervention beyond 12 weeks after the qualifying ischemic event, which was found to have only a minimal effect for secondary stroke prevention [16]. Current updated guidelines therefore recommend that a CEA should ideally be 
performed within 2 weeks of a presenting event [3, 18]. Despite these recommendations, CEA is often delayed for weeks to months [5] due to logistic reasons or a resource shortage, indicating that it might be difficult to translate this ambitious goal into everyday practice on the short term. In a recently published interim-analysis of the GALA Trial, a multicenter randomized trial of general versus local anesthesia during CEA, the median delay between symptoms and surgery was 82 days [4]. Similar time delays between presenting event and CEA have been reported in other population-based surveys across different countries $[7,8,10]$.

Lately, carotid artery stenting (CAS) has increasingly been used as a treatment alternative to CEA, and it is unlikely that the majority of patients are currently treated with CAS within 2 weeks of their symptomatic event. In fact, within the recently published Pro-CAS register and the ICSS study, only about $25 \%$ of the patients had been treated within the recommended time frame of 14 days [9, 19]. In order to increase the benefit of secondary stroke prevention by reducing the time delay between symptoms and carotid revascularization procedures, it is thus crucial to identify the procedural or clinical features that are associated with a delayed intervention. With the knowledge of these factors, further awareness campaigns of the general population can be initiated and a fast-track medical management program can be pursued in patients having a high risk of subsequent stroke.

We used our prospective CAS database to determine which clinical factors were associated with a delayed treatment (i.e., $\geq 14$ days) and which part of the investigation logistics was responsible for this treatment delay.

\section{Methods}

\section{Study population}

From January 2001 to December 2009, a total of 374 patients with symptoms due to a high-grade carotid stenosis were treated with CAS at two university hospitals following a prospective protocol (University of Tübingen $n=220$, University of Göttingen $n=154$ ). Patients were recruited from the in-hospital emergency department where patients presented by themselves or were admitted by the local practitioner or neurologist, the local ambulance, or by admission from regional hospitals. The symptoms of the qualifying event were either classified as an ischemic stroke, an ipsilateral transient ocular, or as a contralateral transient hemispheric cerebral event with respect to the side of the carotid stenosis. In patients with transient symptoms, the qualifying event had resolved completely within $24 \mathrm{~h}$. The symptomatic event had occurred during the last 180 days prior to treatment and patients had to be in clinically stable conditions. All patients had a high-grade carotid stenosis $\geq 70 \%$ as assessed with sonography according to the European guidelines to estimate the degree of stenosis [2] and the grade of stenosis was confirmed angiographically during the stenting procedure.

All patients were informed about the different treatment modalities (CEA, CAS, and best medical treatment) and their specific risks. With respect to the CAS procedure, all patients were informed about the investigational nature of CAS and gave their written informed consent. This study was approved by the local Ethics Review Board.

\section{Data collection}

The following cerebrovascular risk factors were recorded using history or direct measurements: hypertension (blood pressure $\geq 140 / 90 \mathrm{mmHg}$ measured on repeated occasions or presence of antihypertensive drugs), diabetes mellitus (HbAlc $>6.5 \%$, fasting blood glucose $>120 \mathrm{mg} / \mathrm{dl}$, or presence of antidiabetic drugs), hyperlipidemia (fasting serum cholesterol levels $>220 \mathrm{mg} / \mathrm{dl}$ or statin therapy), smoking (current or within the previous year), coronary artery disease (history of angina, myocardial infarction, percutaneous transluminal angioplasty or surgery) and the presence of contralateral carotid disease (as assessed with ultrasound).

The following time intervals were recorded: (1) days between symptom onset and the carotid ultrasound procedure; (2) between symptom onset and hospital admission; (3) the length of the hospital stay; and (4) days between hospital admission and CAS procedure. Time delay to CAS was defined as the period between the first clinical symptoms attributable to the corresponding carotid stenosis and the CAS procedure. A subsequent neurological symptom was scored as an additional recurrent symptom and time intervals were referenced to the day of the first clinical symptom. The duration and the clinical symptoms of the transient symptomatic event and the NIH-SS before the intervention were registered. If a pathological carotid ultrasound finding from an outside hospital or practitioner was verified during hospitalization with locally adopted criteria, the date of the first external ultrasound was recorded for further assessments. All patients were treated by a neurologist with experience in neurovascular diseases. The ABCD score was derived for patients with transient symptoms as follows: age $>60$ years $=1$, elevated blood pressure $(>140 \mathrm{mmHg}$ systolic and/or $>90 \mathrm{mmHg}$ diastolic) $=1$, clinical features of presenting event (unilateral weakness $=2$, speech disturbance without weakness $=1$, other $=0$ ), and duration of clinical symptoms in minutes $(\geq 60=2, \quad 10-59=1,<10=0)$ [17]. One additional 
point was added for the presence of diabetes mellitus to calculate the $\mathrm{ABCD}^{2}$ score [11]. The periprocedural $30 \mathrm{~d}$ complications following CAS were recorded and categorized as minor- (a new neurological deficit that either resolved completely within 7 days or increased the NIH Stroke Scale score by $\leq 3$ ) major stroke (a new neurological deficit that persisted after 7 days and increased the NIH Stroke Scale score by $\geq 4$ ) or death (death of any cause).

\section{Statistical analysis}

Continuous values were expressed as mean \pm standard deviation (SD) and nominal variables as count and percentages, not normally distributed ordinal values as median values with the corresponding interquartile range (IQR), respectively. For comparisons of categorical data, twotailed Chi-square statistics with Yates correction and univariate Fisher's exact test were used. The Fisher's exact test was used when the predicted contingency table cell values were less than five. Ordinal data were compared using a Mann-Whitney $U$ test.

Imbalanced variables with respect to time delay to CAS were identified on the basis of the univariate level if the $p$ value reached a value below 0.1 . A multiple logistic regression analysis was applied to determine the independence of the imbalanced variables. Interaction was assessed by using additive and multiplicative interaction terms. Results are presented as odds ratio with $95 \%$ confidence interval.

A bivariate correlation analysis (Kendall's tau-b) was applied to investigate the interrelation of treatment delay and the year of intervention.

A two-sided $p$ value of less than 0.05 was considered to indicate a statistically significant difference. All statistical analyses were performed using SPSS (Version 17, SPSS inc., Chicago, IL).

\section{Results}

The study population comprised a total of 374 patients (mean age $69.1 \pm 9.2$ years, 269 men, 105 women) with a symptomatic carotid stenosis. For the entire study population, the median time delay between clinical symptoms and CAS was 20 days (IQR 10-37). A total of 153 patients (40.9\%) were treated within 14 days (8, IQR 6-11), whereas $221(59.1 \%)$ patients received their intervention beyond 2 weeks (median: 34, IQR 22-57). A total of 189 $(50.0 \%)$ patients presented with an ischemic stroke and 185 $(49.5 \%)$ with transient symptoms; $143(38.2 \%)$ of these patients had transient hemispherical symptoms and 49 (13.1\%) patients had transient ocular symptoms, respectively.
The baseline characteristics of the patients treated beyond and within 14 days and the results of the univariate analyses are summarized in Table 1.

Patients treated beyond 14 days from symptom onset to CAS received their carotid ultrasound later (29.4 vs. 2.5 days, $p<0.01$ ) and were admitted to the hospital with a significantly longer time delay than patients treated with 14 days ( 37.7 vs. 2.2 days, $p<0.01)$. The time from hospital admission to CAS was also significantly longer in patients treated beyond 14 days (7.7 vs. 5.8 days, $p<0.05$ ).

Patients treated beyond 14 days had a lower NIHSS (2.9 vs. 3.4, $p<0.05)$ and more often had a transient ocular event $(19$ vs. $4.6 \%, p<0.01)$ than patients treated within 14 days, whereas a contralateral occlusion was found more often $(16.3$ vs. $7.2 \%, p<0.01)$ among the early treated patients. The latter patients also had more recurrent neurological symptoms $(11.7$ vs. $5.9 \%, p=0.04)$ before the CAS procedure.

Clinical complication rates after CAS (minor stroke, major stroke, and death within 30 days; 7.8 vs. $8.1 \%$, $p=1.0$ ), as well as hospitalization days (11.7 vs. 11.7, $p=0.94$ ) did not differ between these groups (see Table 1).

In order to calculate the $\mathrm{ABCD}$ and $\mathrm{ABCD}^{2}$ scores in a subgroup of patients with transient symptoms, the duration of the clinical symptoms was divided into three groups $(<10,10-59$ and $\geq 60 \mathrm{~min})$. Within the subgroup of patients with transient symptoms, subjects treated beyond 14 days more frequently had an ocular event than a hemispherical TIA (62.5 vs. $90.4 \%, p<0.01$ ), a lower ABCD and $\mathrm{ABCD}^{2}$ score [3 (IQR 2-4) vs. 4 (IQR 3-4.5), $p<0.01$; 3 (IQR 2-4) vs. 4 (IQR 3-5), $p<0.01]$ and a shorter duration of their initial clinical symptoms $(<10 \mathrm{~min} 64.3$ vs. $32.9 \%$, $p<0.01$ ), whereas subjects with long transient clinical symptoms $(\geq 60 \mathrm{~min})$ were more often treated within 14 days $(41.1$ vs. $17.0 \%, p<0.01)$. The proportion of patients in the subgroup treated beyond 14 days was lower after the year 2004 ( 24.1 vs. $53.4 \%, p<0.01)$.

After applying multiple logistic regression analysis and adjusting for variables that were imbalanced between both groups (contralateral ICA occlusion, hemispherical or ocular TIA, NIH-SS before CAS, recurrent symptomatic event and intervention after the year 2004), a retinal TIA event $(\mathrm{OR}=3.59,95 \%$ CI $1.47-8.74, p<0.01)$ remained the only significant variable that was useful for predicting a delayed treatment beyond 14 days, whereas the year of the intervention $(\mathrm{OR}=0.32,95 \%$ CI $0.20-0.50, p<0.01)$ and a contralateral carotid occlusion $(\mathrm{OR}=0.42,95 \% \mathrm{CI}$ $0.21-0.86, p=0.02$ ) were predictive of an early treatment. Similarly, the year of the intervention $(\mathrm{OR}=0.28,95 \% \mathrm{CI}$ $0.14-0.59, p<0.01$ ) was associated with early treatment, whereas a retinal TIA as the qualifying event $(\mathrm{OR}=6.96$, 95\% CI 2.37-20.47, $p$ value of $p<0.01$ ) was associated with a delayed treatment within the subgroup of patients 
Table 1 Patient characteristics $(n=374)$

\begin{tabular}{|c|c|c|c|}
\hline & \multicolumn{3}{|c|}{ Time delay to intervention } \\
\hline & $<14$ days & $\geq 14$ days & $p$ value \\
\hline$n$ & $153(40.9 \%)$ & $221(59.1 \%)$ & \\
\hline Mean age (years) & $68.8( \pm 9.6)$ & $69.3( \pm 8.9)$ & 0.59 \\
\hline Age $\geq 80$ years & $24(15.7 \%)$ & $29(13.1 \%)$ & 0.48 \\
\hline Male & $115(75.2 \%)$ & $154(70.0 \%)$ & 0.27 \\
\hline Sympt. event-CAS (days) & $8.0( \pm 3.2)$ & $45.8( \pm 34.9)$ & $<0.01$ \\
\hline Sympt. event—carotid ultrasound (days) & $2.5( \pm 2.8)$ & $29.4( \pm 46.8)$ & $<0.01$ \\
\hline Sympt. event—hospital admission (days) & $2.2( \pm 3.2)$ & $37.7( \pm 37.4)$ & $<0.01$ \\
\hline Hospital admission-CAS (days) & $5.8( \pm 3.3)$ & $7.7( \pm 8.6)$ & 0.01 \\
\hline Hospitalization (days) & $11.7( \pm 6.0)$ & $11.7( \pm 8.1)$ & 0.94 \\
\hline \multicolumn{4}{|l|}{ Cerebrovascular risk factors } \\
\hline Hypertension & $130(85.0 \%)$ & $185(83.7 \%)$ & 0.74 \\
\hline Hyperlipidemia & $95(62.1 \%)$ & $135(61.1 \%)$ & 0.84 \\
\hline Tobacco use & $50(32.7 \%)$ & $68(30.8 \%)$ & 0.65 \\
\hline Coronary artery disease & $53(34.6 \%)$ & $62(28.1 \%)$ & 0.33 \\
\hline Diabetes mellitus & $45(29.4 \%)$ & $69(31.2 \%)$ & 0.71 \\
\hline \multicolumn{4}{|l|}{ Lesion characteristics } \\
\hline Degree of stenosis $(\%)$ & $82.0( \pm 9.4)$ & $82.2( \pm 9.0)$ & 0.76 \\
\hline Contralateral ICA occlusion & $25(16.3 \%)$ & $16(7.2 \%)$ & $<0.01^{\mathrm{a}, \mathrm{b}}$ \\
\hline Contralateral ICA stenosis $>70 \%$ & $16(10.5 \%)$ & $23(10.4 \%)$ & 0.76 \\
\hline \multicolumn{4}{|l|}{ Clinical presentation and complications } \\
\hline Ischemic stroke & $80(52.3 \%)$ & $109(49.3 \%)$ & 0.59 \\
\hline Hemispherical TIA & $66(43.1 \%)$ & $70(31.7 \%)$ & $0.02^{\mathrm{a}}$ \\
\hline Retinal TIA & $7(4.6 \%)$ & $42(19.0 \%)$ & $<0.01^{\mathrm{a}, \mathrm{b}}$ \\
\hline NIHSS before CAS & $3.4( \pm 2.6)$ & $2.9( \pm 2.4)$ & $0.02^{\mathrm{a}}$ \\
\hline Recurrent symptoms before CAS & $18(11.7 \%)$ & $13(5.9 \%)$ & $0.04^{\mathrm{a}}$ \\
\hline Intervention $\geq$ year 2005 & $94(61.4 \%)$ & $74(33.5 \%)$ & $<0.01^{\mathrm{a}, \mathrm{b}}$ \\
\hline Periprocedural complications (mmd) & $12(7.8 \%)$ & $18(8.1 \%)$ & 1.00 \\
\hline \multicolumn{4}{|c|}{ Subgroup of patients with transient symptoms $(n=185)$} \\
\hline$n$ & $73(39.5 \%)$ & $112(60.5 \%)$ & \\
\hline Hemispherical TIA & $66(90.4 \%)$ & $70(62.5 \%)$ & $<0.01^{\mathrm{a}}$ \\
\hline Retinal TIA & $7(9.5 \%)$ & $42(37.5 \%)$ & $<0.01^{\mathrm{a}}$ \\
\hline $\mathrm{ABCD}$ score & 4 (IQR 3-4.5) & 3 (IQR 2-4) & $<0.01^{\mathrm{a}}$ \\
\hline $\mathrm{ABCD}^{2}$ score & $4(\mathrm{IQR} 3-5)$ & 3 (IQR 2-4) & $<0.01^{\mathrm{a}}$ \\
\hline Duration of symptoms $\geq 60 \mathrm{~min}$ & $30(41.1 \%)$ & $19(17.0)$ & $<0.01^{\mathrm{a}}$ \\
\hline $10-60 \mathrm{~min}$ & $19(26.0 \%)$ & $21(18.8 \%)$ & 0.24 \\
\hline$<10 \min$ & $24(32.9 \%)$ & $72(64.3 \%)$ & $<0.01^{\mathrm{a}}$ \\
\hline Intervention $\geq$ year 2005 & $39(53.4 \%)$ & $27(24.1 \%)$ & $<0.01^{\mathrm{a}, \mathrm{b}}$ \\
\hline
\end{tabular}

${ }^{\text {a }}$ Factors included in multiple regression analysis

${ }^{\mathrm{b}}$ Factors remained significant after multiple regression analysis mmd minor-, major stroke and death

with transient symptoms after adjustment for imbalanced variables (retinal TIA, $\mathrm{ABCD}$ and $\mathrm{ABCD}^{2}$ score, duration of symptoms $\geq 60$ and $<10 \mathrm{~min}$, year of intervention $>2004$ ). Bivariate correlation analysis revealed a significant negative association between the treatment delay and the year of the CAS intervention (correlation coefficient $=-0.22, p<0.001$, Fig. 1).

\section{Discussion}

In this paper we assessed the time delay between a symptomatic qualifying event due to a high-grade carotid artery stenosis and subsequent CAS treatment in order to identify factors contributing to a deferred stenting procedure which might reduce the benefit of this secondary 


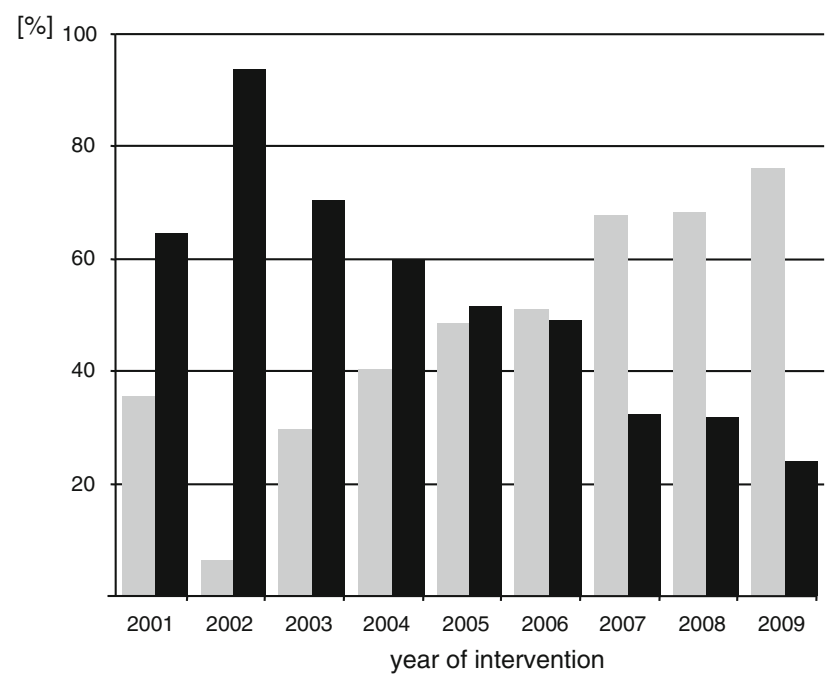

Fig. 1 Proportion of patients treated $<14$ days (grey) and 14 or more days (black) according to the year of the CAS intervention. Within the years there was a significant trend to treat the majority of patients in a more timely fashion (correlation coefficient $=-0.22, p<0.001$ )

stroke prevention. Within this prospective study of two university centers, treatment was delayed $\geq 14$ days in the majority $(59 \%)$ of patients. Independent clinical factors, which were associated with a delayed treatment, were a retinal TIA as a qualifying event and a treatment before the year 2005, whereas the presence of a contralateral carotid occlusion was significantly associated with an early treatment. Similar results were found for the subgroup of patients who had presented with transient symptoms. Finally, the presence of a high $\mathrm{ABCD}$ - or of a high $\mathrm{ABCD}^{2}$ score did not influence the time delay to treatment.

Large artery atherosclerosis is the most meaningful cause for early stroke recurrence after a TIA or minor stroke [13]. This finding is thought to be caused by an ongoing embolic process of the carotid artery originating from a ruptured vulnerable plaque. Early carotid surgery is not associated with a higher rate of periprocedural complications [16] than delayed surgery in clinical stable patients. Similarly, and in good agreement with our current results, large registries indicate that the timing of CAS does not influence the outcome [19]. Moreover, the benefit of a delayed CEA treatment vanishes over time and may even be absent for certain patient subgroups if carried out beyond 3 months [16]. Therefore, current guidelines recommend an early carotid revascularization (ideally within 14 days) in patients with a recently symptomatic carotid stenosis $[3,18]$. However, despite these recommendations, numerous patients are currently still being treated with either CEA or CAS beyond the recommended 14-day time interval $[4,5,7,8,10]$, indicating a possible shortage of resources or difficulties to implement these guidelines into daily routine. In this study, many patients were also treated beyond the proposed 14-day time frame with CAS. This finding is in line with recently published data of the ProCAS registry [19]. In fact, only $26 \%$ (609 of 2,344) of patients with a symptomatic carotid artery stenosis were treated within 14 days within this prospective multicenter registry. Likewise, within the lately published ICSS study in which the efficacy of CAS or CEA for treating a symptomatic carotid stenosis was randomly investigated, only $25 \%$ of patients in the CAS and $18 \%$ in the CEA group were treated within 14 days [9].

To obtain a faster treatment in the future, our data corroborate the need for an accelerated admittance strategy. As delineated in Table 1, most of the treatment delay was due to either the delayed referral to an ultrasound examination of the carotid arteries or to the hospital admittance itself. In contrast, the times from hospital admittance to CAS, as well as the total hospitalization times did not have a major impact on a delayed treatment. The in-hospital patient management only marginally contributes to the delayed treatment, which might for instance be attributable to a risk of reperfusion hemorrhages in patients with a high-grade carotid stenosis. Although we are unable to differentiate between patient-related and non-patient-related factors contributing to the pre-hospitalization times, it is likely that a combination of these factors leads to a delayed referral and thus treatment in many patients. On the one hand, patients themselves, especially those with a TIA, may delay seeking medical attention. At least indirectly, this assumption is supported by our observation that patients with more severe transient or permanent neurological deficits were treated in a more timely fashion than those for instance with an isolated amaurosis fugax.

On the other hand, patients often primarily present to their local practitioners, who might delay referral to the hospital or to physicians with expertise in carotid ultrasound. As outlined in Fig. 1, there was an ongoing rise of the proportion of patients being treated early, reaching nearly $80 \%$ in the year 2009. Therefore, it seems to be possible to treat the vast majority of patients in a timely fashion and that this has been increasingly implemented during the last years.

A contralateral ICA occlusion was significantly associated with a treatment within 14 days. This may be related to earlier results of the NASCET-data in which these patients carried a very high risk for further strokes [1]. At least within our patient cohort this fact seems to have already been integrated into daily practice.

The usefulness of the $\mathrm{ABCD}$ and $\mathrm{ABCD}^{2}$ score in patients with transient symptoms could recently be shown to predict the individual patient risk for subsequent strokes $[11,17,20]$. Although a higher score was found more frequently in the group of patients treated within 2 weeks, $>40 \%$ of patients with an $\mathrm{ABCD}$ or $\mathrm{ABCD}^{2}$ score $\geq 4$ were 
treated beyond this crucial time frame. Taking into account the proposed high risk for stroke recurrence in patients with transient symptoms due to a carotid stenosis and high $\mathrm{ABCD} / \mathrm{ABCD}^{2}$ scores [12], these results clearly support the notion that further awareness campaigns for the general population, as well as for general practitioners need to be initiated.

We acknowledge that our study has several limitations. Despite the fact that these data were acquired in a prospective manner, parts of the analysis were done in a retrospective fashion. Although primary outcome parameters were not imbalanced between the centers, it is not possible to definitely rule out a potential selection bias of slightly different CAS protocols, patient selection, admittance practices, and patient management during the pre-hospitalization phase.

In conclusion, in this study we could show that many patients with a symptomatic carotid stenosis are currently still being treated with CAS beyond the recommended time frame of 2 weeks after their presenting ischemic event. In addition to a delayed admittance to the hospital, treatment delay was most pronounced in patients with an amaurosis fugax, whereas a contralateral carotid occlusion led to an early intervention. Although CAS was performed more quickly in the last years, there is still scope for an even more accelerated treatment strategy, which might prevent recurrent strokes prior to treatment.

Conflict of interest A.K. has received speaker honoraria from Boehringer Ingelheim. K.G., S.S., K.W., S.M.P.P., U.E. and M.K. have no conflicts of interest to disclose.

Open Access This article is distributed under the terms of the Creative Commons Attribution Noncommercial License which permits any noncommercial use, distribution, and reproduction in any medium, provided the original author(s) and source are credited.

\section{References}

1. (1991) Beneficial effect of carotid endarterectomy in symptomatic patients with high-grade carotid stenosis. North American Symptomatic Carotid Endarterectomy Trial Collaborators. N Engl J Med 325:445-453

2. (1998) Randomised trial of endarterectomy for recently symptomatic carotid stenosis: final results of the MRC European Carotid Surgery Trial (ECST). Lancet 351:1379-1387

3. (2008) Guidelines for management of ischaemic stroke and transient ischaemic attack 2008. Cerebrovasc Dis 25:457-507

4. Dellagrammaticas D, Lewis S, Colam B, Rothwell PM, Warlow CP, Gough MJ (2007) Carotid endarterectomy in the UK: acceptable risks but unacceptable delays. Clin Med 7:589-592

5. Fairhead JF, Mehta Z, Rothwell PM (2005) Population-based study of delays in carotid imaging and surgery and the risk of recurrent stroke. Neurology 65:371-375

6. Giles MF, Rothwell PM (2007) Risk of stroke early after transient ischaemic attack: a systematic review and meta-analysis. Lancet Neurol 6:1063-1072
7. Gladstone DJ, Oh J, Fang J, Lindsay P, Tu JV, Silver FL, Kapral MK (2009) Urgency of carotid endarterectomy for secondary stroke prevention: results from the Registry of the Canadian Stroke Network. Stroke 40:2776-2782

8. Halliday AW, Lees T, Kamugasha D, Grant R, Hoffman A, Rothwell PM, Potter JF, Horrocks M, Naylor R, Rudd AG (2009) Waiting times for carotid endarterectomy in UK: observational study. BMJ 338:b1847

9. International Carotid Stenting Study investigators, Ederle J, Dobson J, Featherstone RL, Bonati LH, van der Worp HB, de Borst GJ, Lo TH, Gaines P, Dorman PJ, Macdonald S, Lyrer PA, Hendriks JM, McCollum C, Nederkoorn PJ, Brown MM (2010) Carotid artery stenting compared with endarterectomy in patients with symptomatic carotid stenosis (International Carotid Stenting Study): an interim analysis of a randomised controlled trial. Lancet 375:985-997

10. Johansson EP, Wester P (2008) Delay from symptoms to carotid endarterectomy. J Intern Med 263:404-411

11. Johnston SC, Rothwell PM, Nguyen-Huynh MN, Giles MF, Elkins JS, Bernstein AL, Sidney S (2007) Validation and refinement of scores to predict very early stroke risk after transient ischaemic attack. Lancet 369:283-292

12. Koton S, Rothwell PM (2007) Performance of the ABCD and $\mathrm{ABCD} 2$ scores in TIA patients with carotid stenosis and atrial fibrillation. Cerebrovasc Dis 24:231-235

13. Lovett JK, Coull AJ, Rothwell PM (2004) Early risk of recurrence by subtype of ischemic stroke in population-based incidence studies. Neurology 62:569-573

14. Ois A, Cuadrado-Godia E, Rodriguez-Campello A, JimenezConde J, Roquer J (2009) High risk of early neurological recurrence in symptomatic carotid stenosis. Stroke 40:2727-2731

15. Purroy F, Montaner J, Molina CA, Delgado P, Ribo M, AlvarezSabin J (2007) Patterns and predictors of early risk of recurrence after transient ischemic attack with respect to etiologic subtypes. Stroke 38:3225-3229

16. Rothwell PM, Eliasziw M, Gutnikov SA, Warlow CP, Barnett HJ (2004) Endarterectomy for symptomatic carotid stenosis in relation to clinical subgroups and timing of surgery. Lancet 363:915-924

17. Rothwell PM, Giles MF, Flossmann E, Lovelock CE, Redgrave JN, Warlow CP, Mehta Z (2005) A simple score (ABCD) to identify individuals at high early risk of stroke after transient ischaemic attack. Lancet 366:29-36

18. Sacco RL, Adams R, Albers G, Alberts MJ, Benavente O, Furie K, Goldstein LB, Gorelick P, Halperin J, Harbaugh R, Johnston SC, Katzan I, Kelly-Hayes M, Kenton EJ, Marks M, Schwamm LH, Tomsick T (2006) Guidelines for prevention of stroke in patients with ischemic stroke or transient ischemic attack: a statement for healthcare professionals from the American Heart Association/American Stroke Association Council on Stroke: cosponsored by the Council on Cardiovascular Radiology and Intervention: the American Academy of Neurology affirms the value of this guideline. Stroke 37:577-617

19. Theiss W, Hermanek P, Mathias K, Bruckmann H, Dembski J, Hoffmann FJ, Kerner R, Leisch F, Mudra H, Schulte KL, Sievert $\mathrm{H}$ (2008) Predictors of death and stroke after carotid angioplasty and stenting: a subgroup analysis of the Pro-CAS data. Stroke 39:2325-2330

20. Tsivgoulis G, Stamboulis E, Sharma VK, Heliopoulos I, Voumvourakis K, Teoh HL, Patousi A, Andrikopoulou A, Lim EL, Stilou L, Sim TB, Chan BP, Stefanis L, Vadikolias K, Piperidou C (2010) Multicenter external validation of the ABCD2 score in triaging TIA patients. Neurology 74:1351-1357 